\title{
Laser photoacoustic spectroscopy applications in breathomics
}

\author{
Yury V. Kistenev ${ }^{1,2^{*}}$, Alexey V. Borisov ${ }^{1,2}$, Victor V. Nikolaev ${ }^{1,3}$, Denis A. Vrazhnov ${ }^{1,3}$, \\ and Dmytry A.Kuzmin ${ }^{2}$ \\ ${ }^{1}$ Tomsk State University, Tomsk 634050, Russia \\ 2 Siberian State Medical University, Tomsk 634050, Russia \\ ${ }^{3}$ Institute of Strength Physics and Materials Science of Siberian Branch of the RAS, Tomsk 634055, Russia \\ * e-mail: yv.kistenev@gmail.com
}

\begin{abstract}
The breathomics approach to express-diagnosis of bronchopulmonary diseases based on spectral analysis of volatile organic compounds in a patient's exhaled air is discussed. The basic demands and possible technical solutions to laser photoacoustic spectroscopy equipment in a framework of breathomics are presented. An example of differential diagnostics of the set of bronchopulmonary diseases, including lung cancer (LC) patients $(\mathrm{N}=9)$; patients with chronic obstructive pulmonary disease (COPD) $(\mathrm{N}=12)$; patients with pneumonia $(\mathrm{N}=11)$ and a control group of healthy volunteers using breath air analysis by laser photoacoustic spectroscopy and machine learning is presented. (C) 2019 Journal of Biomedical Photonics \& Engineering.
\end{abstract}

Keywords: Breathomics; exhaled air analysis; laser photoacoustic spectroscopy; optical parametric oscillator; machine learning; lung cancer.

Paper \#3307 received 25 Nov 2018; accepted for publication 20 Mar 2019; published online 28 Mar 2019. doi: 10.18287/JBPE19.05.010303.

\section{Introduction}

Control of metabolites in exhaled air produced by biochemical reactions in cells and organs being called as "breathomics" provides ability of express and noninvasive medical screening. Typical metabolites, produced by pathological processes in the body, include:

— inorganic substances, e.g., carbon dioxide, oxygen, and nitric oxide;

— non-volatile substances, e.g., isoprostanes, cytokines, leukotrienes and hydrogen peroxide;

— volatile organic compounds (VOCs), which include saturated hydrocarbons (ethane, pentane, aldehydes), unsaturated hydrocarbons (isoprene), oxygen containing (acetone), sulphur containing (ethyl mercaptane, dimethylsulfide) and nitrogen containing (dimethylamine, ammonia) [1].

In spite of gas chromatography is a "gold standard" for content analysis of biological origin gas mixtures, this method is quite complicated for routine medicine use.

A set of electrochemical sensors, each of which corresponds to a particular substance (called by "electronic nose" or "e-nose") is one of instrumental methods of exhaled air VOCs control, being suitable for routine measurements. For example "Cyranose 320" includes 32 polymer chemiresistors [2]. The main disadvantages of contact sensors are low selectivity and fast degradation due to surface contamination.

IR laser absorption spectroscopy (LAS) has high sensitivity of molecular species control on the level of $\mathrm{ppb}$ and below, as they frequently occur in environmental, medical or biological applications [3]. Most sensitive detection provides photoacoustic spectroscopy, Faraday modulation spectroscopy, cavity ring-down spectroscopy (CRDS) or cavity leak-out spectroscopy (CALOS) which is a $\mathrm{CW}$ version of CRDS [3].

Faraday modulation spectroscopy is suitable only for detection of paramagnetic species in gas phase. A significant limitation of the CRDS is technical complexity of wavelength tuning, because this method is based on using of a resonator with high reflected mirrors, but a high reflection is achieved only in a narrow spectral range [4].

Laser photoacoustic spectroscopy (LPAS) is based on registration of pressure wave which is generated in 
an analyzed gas sample due to absorption of amplitude modulated laser beam tuned on a frequency of resonant absorption of a specific substance of the sample. Some examples of breathomics LPAS applications are presented below.

In lipid peroxidation, reactive oxygen species react with polyunsaturated fatty acids to form a variety of products, including pentane, ethane, and ethylene (ET). ET concentration was monitored in real time by using a LPAS gas analyzer [5]. The gas analyzer consists of a line-tunable $\mathrm{CO}_{2}$ laser and a photoacoustic detector (PAD). A sodalime and a $\mathrm{KOH}$ scrubbers were used to remove $\mathrm{CO}_{2}$ from the air sample. $\mathrm{A} \mathrm{CaCl}_{2}$ scrubber was used to decrease the water content. Volatile gases were removed using a liquid nitrogen-cooled cryogenic trap $(125 \mathrm{~K})$. The $\mathrm{CO}_{2}$ laser lines $10 \mathrm{P}(14)\left(\lambda=948.48 \mathrm{~cm}^{-1}\right)$ and $10 \mathrm{P}(12) \quad\left(\lambda=951.19 \mathrm{~cm}^{-1}\right)$ were used for ET concentration measurement. This system has ET detection limit of 6 ppt.

The levels of ET and ammonia in exhaled breath samples (EBS) from patients with cancer and renal failure were measured at sub $\mathrm{ppb}$ concentration sensitivities using $\mathrm{CO}_{2}$ LPAS analyzer [6]. The ET concentration was measured on $10 \mathrm{P}(14) \mathrm{CO}_{2}$ laser line, where the ET absorption coefficient is maximal $\left(30.4 \mathrm{~cm}^{-1} \mathrm{~atm}^{-1}\right)$, the ammonia concentration was measured on $9 \mathrm{R}(30) \mathrm{CO}_{2}$ laser line, where the ammonia absorption coefficient is $57 \mathrm{~cm}^{-1} \mathrm{~atm}^{-1}$.

LPAS sensitivity can be improved using PAD intracavity placement [7]. The $\mathrm{C}_{2} \mathrm{H}_{4}$ detection limit by $\mathrm{CO}_{2}$ laser intracavity PAD cell in purified from other hydrocarbons ambient air was about 6 ppt $[8,9]$.

The sensitivity of LPAS can be essentially increased, when the laser beam is modulated by a resonance frequency of the photoacoustic cell. It this case the generated photoacoustic signal is amplified by the quality factor (Q-factor) of the acoustic resonance, which can be achieved up to several hundred [10].

The most frequently used types of resonant LPAS detectors are based on Helmholtz resonators, onedimensional cylindrical resonators and cavity resonators $[11,12]$. The example of construction and sensitivity curve of PAD with two Helmholtz resonators is presented in the Fig. 1 [13].

LPAS gas analyzer with tunable $\mathrm{CO}_{2}$ laser and intracavity PAD with two Helmholtz resonators was used to measure the spectral characteristics of EAS of the following groups of patients [7]: healthy participants (control group), patients with a bronchopulmonary disease (chronic obstructive pulmonary disease (COPD), asthma, pneumonia), patients with a nonbronchopulmonary disease (coronary heart disease, gastric ulcer, duodenal ulcer), patients with tuberculosis. In total, the quantity of participants was about 200 .

The comparison of measured absorption spectra of EBS for a group under study $S$ was carried out in terms of Mahalanobis distance relatively to the reference group $S_{0}$. Let mark measured set of absorption coefficients of the EBS spectra for a group under study $S$ as $\overrightarrow{y_{j}}, j=\overline{1, N_{S}}$ and the same values for the reference group as $\overrightarrow{x_{i}}, i=\overline{1, N_{S_{0}}}$, correspondently. Here, sets $\vec{x}_{j}, \vec{y}_{j}$ can be considered as feature vectors of the state, $N_{S}$ and $N_{S_{0}}$ are the total quantity of measured spectra of EAS in the every group. So, the average square of the Mahalanobis distance (ASMD) can be defined as

$$
\operatorname{ASMD}\left(\vec{y}_{j}\right)=\frac{1}{2 m N_{S_{0}}} \sum_{i=1}^{N_{S_{0}}} d_{M}^{2}\left(\vec{y}_{j}, \vec{x}_{i}\right)
$$

where $\quad d_{M}(\vec{x}, \vec{y})=\sqrt{(\vec{x}-\vec{y})^{T} C^{-1}(\vec{x}-\vec{y})} \quad$ is the Mahalanobis distance, $C$ is the covariance matrix of the feature vectors for participants from the reference group $S_{0}, m$ is the dimension of the feature space.

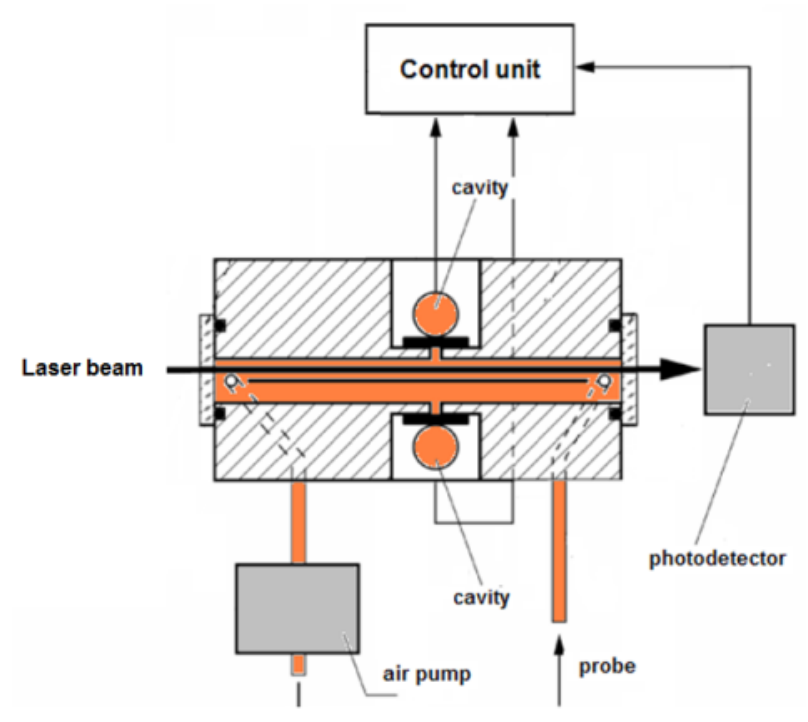

a

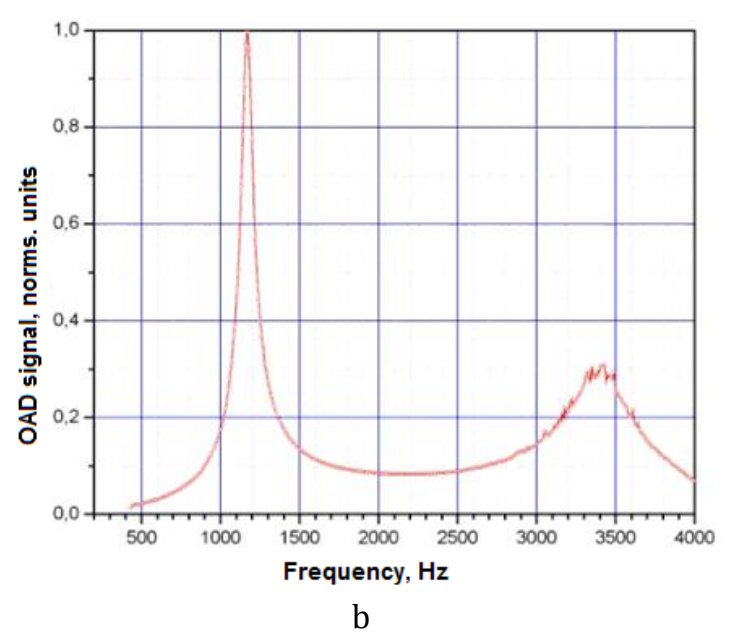

Fig. 1 The design of PAD with two Helmholtz resonators (a) and its sensitivity dependency on the frequency of amplitude modulation of the laser source (b). 
In the Fig. 2 the set of absorption coefficients of EBS from patients with tuberculosis is used as feature vectors $\vec{x}_{i}$ of the reference group $S_{0}$, the set of absorption coefficients of EBS from other participants are used as feature vectors $\vec{y}_{j}$ of the group $S$. The AVMD of EBS absorption spectrum in the 10P and 10R spectral bands of $\mathrm{CO}_{2}$ laser generation for participants are marked in the Fig. 2 as "integral estimations 1" and "integral estimations 2", correspondently [7].

The photoacoustic signal is proportional to absorbed volume fraction of input light source energy. Therefore, a high-power light sources are preferable. Light sources that have been used in photoacoustic spectroscopy include broadband infrared radiation sources, that is, black-body radiators and light-emitting diodes; but in most cases various lasers $\left(\mathrm{CO}_{2}, \mathrm{CO}\right.$, diode, quantum cascade, and Nd:YAG lasers) are used [14].

Optical parametric oscillator (OPO) systems provide light power from few $100 \mathrm{~mW}$ to more than $1 \mathrm{~W}$ in the wavelength range of 2-4 $\mu \mathrm{m}$. OPOs were first used in photoacoustic detection of organic compounds near 3.3 $\mu \mathrm{m}$ at ppm-level and successfully applied later to measure formaldehyde with $\mathrm{ppb}$ and ethane with subppb accuracy [15].

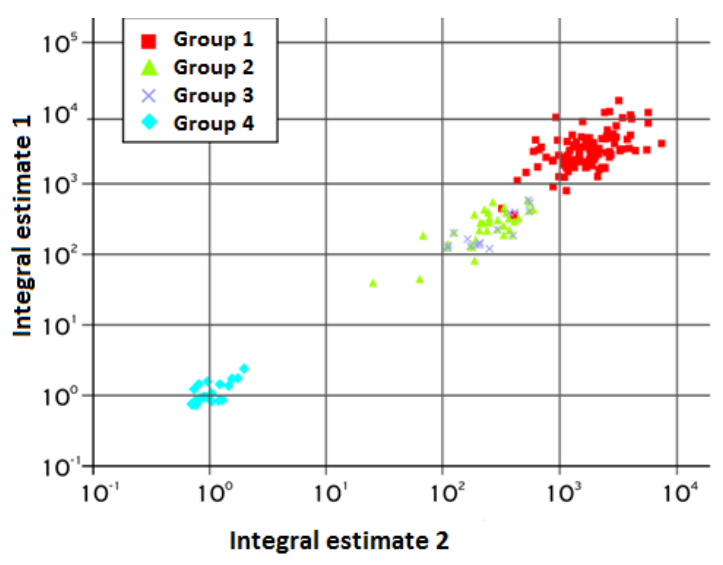

Fig. 2 Distribution of point estimates of absorption spectra of exhaled air. The Group 1 includes healthy participants, the Group 2 includes patients with bronchopulmonary diseases (COPD, asthma, pneumonia), the Group 3 includes nonbronchopulmonary diseases (coronary heart disease, gastric ulcer, duodenal ulcer), the Group 4 includes patients with tuberculosis.

An IR gas sensor combining a continuous-wave (CW) OPO with Cavity Leak-Out spectroscopy (CALOS) was presented [3]. The PPLN nonlinear crystal (19 mm long) contains 19 gratings with poling periods between $28.64 \mu \mathrm{m}$ and $30.16 \mu \mathrm{m}$ and is mounted inside a self designed oven for operation at temperatures between 150 and $200{ }^{\circ} \mathrm{C}$. The temperature stability is $8 \mathrm{mK}$ (standard deviation) over 4 hours. The first crystal surface is HR coated for both pump (94.3\%) and signal $(99.9 \%)$ waves thus serving as the common first mirror for both resonators. The PPLN crystal was pumped at $1064 \mathrm{~nm}(2 \mathrm{~W})$. Dual cavity design in OPO was used for wavelength selection within the tuning range of the OPO $(3.1-3.8 \mu \mathrm{m})$. CALOS cell was based on a high finesse optical resonator $(\mathrm{L}=52.5 \mathrm{~cm})$ formed by two highly reflective mirrors $(\mathrm{R}=99.985 \%$, Los Gatos). A noise-equivalent absorption coefficient of $1.6 \cdot 10-10 \mathrm{~cm}^{-1} / \sqrt{\mathrm{Hz}}$ was achieved for integration times up to $180 \mathrm{sec}$. This corresponds to a detection limit for ethane at sub-ppt level.

The OPO-based spectrometer with PAD (wavelength near $3 \mu \mathrm{m}, 8 \mathrm{MHz}$ linewidth, output power $\sim 1 \mathrm{~W}$ ) was used for the detection of hydrogen cyanide (HCN) emission from clover leaves, and Pseudomonas bacteria; in addition, the presence of $\mathrm{HCN}$ in exhaled human breath was measured [16]. For specific experiments, the spectrometer is operated continuously up to 10 days and has a detection limit of $0.4 \mathrm{ppbv}$ of $\mathrm{HCN}$ in air over $10 \mathrm{~s}$, using the P8 rotational line in the $v_{3}$ vibrational band of $\mathrm{HCN}$ at $3287.25 \mathrm{~cm}^{-1}$. This provided an overall sensitivity of the system of $2.5 \times 10^{-9} \mathrm{~cm}^{-1} \mathrm{~Hz}^{-1 / 2}$.

Table 1 Technical characteristics of the spectrometer LaserBreeze [17].

\begin{tabular}{lc}
\hline \multicolumn{1}{c}{ Parameter } & Value \\
\hline Source of radiation & $\begin{array}{c}\text { Optical parametric } \\
\text { oscillator }\end{array}$ \\
\hline The spectral tuning range & $2.5-10.7 \mu \mathrm{m}$ \\
\hline $\begin{array}{l}\text { Concentration detection } \\
\text { sensitivity, not worse }\end{array}$ & $1 \mathrm{ppb}$ \\
\hline $\begin{array}{l}\text { The number of detected } \\
\text { substances, not less }\end{array}$ & 20 \\
\hline $\begin{array}{l}\text { The relative error in measuring } \\
\text { of the concentration of } \\
\text { biomarkers, not more }\end{array}$ & $30 \%$ \\
\hline $\begin{array}{l}\text { The accuracy and selectivity of } \\
\text { biomarkers detection, not less }\end{array}$ & $95 \%$ \\
\hline $\begin{array}{l}\text { The volume of the analyzed gas } \\
\text { samples for registration of the } \\
\text { composition and concentration of } \\
\text { biomarkers, not more }\end{array}$ & $50 \mathrm{~cm}^{3}$ \\
\hline $\begin{array}{l}\text { Time of registration of one } \\
\text { biomarker in the sample, not } \\
\text { more }\end{array}$ & $3 \mathrm{~s}$ \\
\hline $\begin{array}{l}\text { Time of registration of 10 } \\
\text { biomarkers in the sample, not } \\
\text { more }\end{array}$ & $2 \mathrm{~min}^{2}$ \\
\hline
\end{tabular}

We used developed by Special technologies Ltd. laser photoacoustic gas analyzer LaserBreeze based on the dual OPO with tuning range 2.5-10.7 $\mu \mathrm{m}$ [17]. Two types of nonlinear elements are used in this OPO: periodically poled lithium niobate structure (PPLN) and mercury thiogallate crystal $\mathrm{HgGa}_{2} \mathrm{~S}_{4}$ (HGS). Nd:YLF laser (10 ns, $0.5-1.5 \mathrm{kHz}, 1.5 \mathrm{~mJ}$ ) was used as a pump source. The linewidth of developed OPOs was $3-4 \mathrm{~cm}^{-1}$. The average power of OPO based on PPLN structure was $20 \mathrm{~mW}(1700 \mathrm{~Hz})$. The average power of OPO 
Table 2 The binary "One-vs-One” SVM classification quality of the groups under study.

\begin{tabular}{|c|c|c|c|c|c|c|}
\hline \multirow{2}{*}{ Groups } & \multirow{2}{*}{$\begin{array}{c}\text { Kernel } \\
\text { parameter }\end{array}$} & \multirow{2}{*}{$\begin{array}{l}\text { Principal } \\
\text { components }\end{array}$} & \multicolumn{2}{|c|}{ Sensitivity } & \multicolumn{2}{|c|}{ Specificity } \\
\hline & & & Mean & Dispersion & Mean & Dispersion \\
\hline LC- Pneumonia & 0.7152 & 2 and 6 & 0.96 & 0.0014 & 0.93 & 0.0012 \\
\hline LC- COPD & 1.2216 & 2 and 6 & 0.98 & 0.0003 & 0.94 & 0.0007 \\
\hline $\begin{array}{c}\text { LC - healthy } \\
\text { volunteers }\end{array}$ & 0.2698 & 1 and 5 & 0.96 & 0.0011 & 0.90 & 0.0013 \\
\hline $\begin{array}{c}\text { COPD- } \\
\text { Pneumonia }\end{array}$ & 1.2041 & 2 and 5 & 0.95 & 0.0016 & 0.95 & 0.0012 \\
\hline $\begin{array}{c}\text { COPD-healthy } \\
\text { volunteers }\end{array}$ & 1.2414 & 1 and 5 & 0.86 & 0.0022 & 0.83 & 0.0020 \\
\hline $\begin{array}{l}\text { Pneumonia- } \\
\text { healthy } \\
\text { volunteers }\end{array}$ & 0.5641 & 2 and 6 & 0.96 & 0.0009 & 0.92 & 0.0019 \\
\hline
\end{tabular}

based on HGS crystal was $9 \mathrm{~mW}(900 \mathrm{~Hz})$. The double channel resonant photo-acoustic cell was used for recording absorption spectra of gaseous samples. Technical characteristics of the LaserBreeze gas analyzer are shown in the Table 1.

The aim of the paper is to show abilities of laser photoacoustic gas analyzer based on OPO with extra wide tuning range for breathomics.

\section{Materials and methods}

The protocol of the research was approved by the Ethic Committee of the Siberian State Medical University (Tomsk, Russia), Ref. Number 2882 at 24.11.2011. All participants were preliminary informed about details of research and signed "Informed agreement" on the actions carried out. The interaction with the patients was limited by the sampling of a part of exhaled air into a disposable container.

The sampling procedure was carried out before eating or 2 hours thereafter. Before sampling, participants rinsed the mouth with water without any oral cavity cleaning agents. Then participant did some calm breaths through a sterile plastic tube into the sample container (syringe). The "dead volume" was exhaled outside the container.

The study involved four groups: patients with bronchopulmonary diseases including lung cancer (LC) patients $(\mathrm{N}=9)$; patients with chronic obstructive pulmonary disease (COPD) $(\mathrm{N}=12)$; patients with pneumonia $(\mathrm{N}=11)$ and a control group of healthy volunteers $(\mathrm{N}=29)$. All patients had been treated in medical organizations, so the diagnosis has been verified by clinical methods.

Exhaled breath samples were collected in $150 \mathrm{ml}$ disposable plastic containers (syringe) and analyzed using the LaserBreeeze gas analyzer. All measurements were carried out at room temperature $\left(20-25^{\circ} \mathrm{C}\right)$ and humidity $(50-60 \%)$. We repeat measurements of the EAS absorption spectrum 5 times.

The first step in data analysis was consisted in informative features extraction. The principal component analysis (PCA) is one of the most effective methods in this field. The basic idea of PCA is to transform data to reduced number of new orthogonal variables, termed the principal components [18].

Often, the final goal of breathomics analysis consists in predictive model construction, which can be used for further diagnostics. One of the most efficient approaches to this task is machine learning methods usage, including supervised and unsupervised learning. Usually, the classification model a based on using supervised learning methods. These methods are based on using of a part of experimental data for training of algorithm of classification and other - for testing.

Below we used the support vector machine (SVM) method for classification. SVM provides binary classification and it is based on building up the maximum-width stripe, which spatially separate groups under study. The algorithm is based on analysis of scalar product of the feature vectors. When the building of such stripe is impossible, the kernel transform can help to provide classification, which is based on analysis of scalar product of the feature vectors functions [19].

\section{Analysis of the experimental data}

Initially we construct all variants of the "One-vs-One" binary classifies between groups under study, using SVM approach with radial basis function (RBF) kernel. First fifteen principal components were used for SVM classification. The random splitting of initial data on teaching and testing sets was repeated 250 times. The optimal kernel parameters had been evaluated using machine learning approach. The Table 2 shows the 
Table 3. Results of differential diagnosis of the groups under study

\begin{tabular}{|c|c|c|c|c|c|c|}
\hline \multirow{3}{*}{ Group } & \multicolumn{6}{|c|}{ Diagnosis } \\
\hline & \multicolumn{2}{|c|}{ Set right } & \multicolumn{2}{|c|}{ Set wrong } & \multicolumn{2}{|c|}{ Did not set } \\
\hline & Mean & Dispersion & Mean & Dispersion & Mean & Dispersion \\
\hline $\mathrm{LC}$ & 0.9565 & 0.0013 & 0.0341 & 0.0011 & 0.0094 & 0.0013 \\
\hline COPD & 0.8112 & 0.0091 & 0.0981 & 0.0082 & 0.0907 & 0.0047 \\
\hline Pneumonia & 0.8412 & 0.0048 & 0.0991 & 0.0032 & 0.0597 & 0.0025 \\
\hline $\begin{array}{c}\text { Healthy } \\
\text { volunteers }\end{array}$ & 0.8946 & 0.0038 & 0.0901 & 0.0024 & 0.0153 & 0.0018 \\
\hline
\end{tabular}

results of the binary "One-vs-One" classification in terms of the specificity and sensitivity.

These "One-vs-One" classifiers allow one to construct differential diagnosis rules. One of the possible approaches to this task is enumeration of these classifiers for a feature vector of an object under study [20]. Below the differential diagnostics rule was based on the result of "One-vs-One" classifications, which was appeared more times. Diagnosis did not set, if several results of classification (LC-COPD-HealthyPneumonia) for definite feature vector from the testing set met the same number of times. The approach was applied to various splitting of the initial data training and testing data sets, while a variation of the average value of accuracy of each diagnosis within the last 20 iterations exceeded 1\%. Results of differential diagnosis are presented in table 3 .

\section{Conclusion}

Breathomics is a promising tool for fast and noninvasive medical diagnosis. LPAS is one of the most sensitive and universal methods of VOCs detection in a breath air.

The ability of LPAS technique significantly depends on a range of the used laser source spectral tuning. The presented data of breath air analysis using IR laser photoacoustic spectrometer LaserBreeze based on the dual optical parametric oscillator with extra-wide spectral tuning range shows its high potential in diagnostics of bronchopulmonary diseases. The "profiling" approach, based on of the set of markers control or profile of the absorption spectrum of breath sample as a "fingerprint" of the state is presented. The accuracy of multiclass classification using a set of binary SVM “One-vs-One" classifiers based on spectral analysis of exhaled air of patients is high enough for using in routine practices, especially for screening tests.

On the whole, the future of breathomics practical use demands cost-effective and informative measurement equipment, standardization of the sampling, discovery of most specific biomarkers/profiles of biomarkers, effective methods of data analysis.

\section{Disclosures}

All authors declare that there is no conflict of interests in this paper.

\section{Acknowledgments}

The presented results were carried out with partial financial support of the FCPIR Contract No. 14.578.21.0082 (ID RFMEFI57814X0082). This work was also performed within the frame of the Fundamental Research Program of the State Academies of Sciences for 2013-2020, line of research III.23.

\section{References}

1. A. G. Dent, T. G. Sutedja, and P. V. Zimmerman, "Exhaled breath analysis for lung cancer," Journal of Thoracic Disease 5, 540-550 (2013).

2. M. P. Fernandes, S. Venkatesh, and B. G. Sudarshan, "Early Detection of Lung Cancer Using Nano-Nose - A Review," Open Biomedical Engineering Journal 9(1), 228-233 (2015).

3. F. Kühnemann, F.Müller, G. von Basum, D. Halmer, A. Popp, S. Schiller, P. Hering, and M. Muertz, "CW-OPO based cavity-leak-out spectrometer for ultra-sensitive and selective mid infrared trace gas analysis," Proceedings of SPIE 5337, 117-127 (2004).

4. V. Nikolaev, V. N. Ochkin, and S. N. Tskhai, "Fast recording of weak absorption spectra in optical cavity using tunable laser," Laser Physics Letters 10(11), 115701 (2013). 
5. H. W. A. Berkelmans, B. W. M. Moeskops, J. Bominaar, P. T. J. Scheepers, and F. J. M. Harren, "Pharmacokinetics of ethylene in man by on-line laser photoacoustic detection," Toxicology and Applied Pharmacology 190(3), 206-213 (2003).

6. C. Popa, A. M. Bratu, C. Matei, R. Cernat, A. Popescu, and D. C. Dumitras, "Qualitative and Quantitative Determination of Human Biomarkers by Laser Photoacoustic Spectroscopy Methods," Laser Physics 21(7), 13361342 (2011).

7. Y. V. Kistenev, A. A. Karapuzikov, "Methods of spectral analysis of exhaled air suitable for routine diagnostics of diseases of the respiratory system," Advanced biomaterials and devices in biomedicine 2, 79-87 (2015).

8. J. A. de Gouw, S. Te Lintel Hekkert, J. Mellqvist, C. Warneke, E. L. Atlas, F. C. Fehsenfeld, A. Fried, G. J. Frost, F. J. M. Harren, J. S. Holloway, B. Lefer, R. Lueb, J. F. Meagher, D. D. Parrish, M. Patel, L. Pope, D. Richter, C. Rivera, T. B. Ryerson, J. Samuelsson, J. Walega, R. A. Washenfelder, P. Weibring, and X. Zhu, "Airborne Measurements of Ethene from Industrial Sources Using Laser Photo-Acoustic Spectroscopy," Environmental Science \& Technology 43 (7), 2437-2442 (2009).

9. F. G. C. Bijnen, J. Reuss, and F. J. M. Harren, "Geometrical optimization of a longitudinal resonant photoacoustic cell for sensitive and fast trace gas detection," Review of Scientific Instruments 67(8), 2914 (1996).

10. C.-M. Lee, K. V. Bychkov, V. A. Kapitanov, A. I. Karapuzikov, Y. N. Ponomarev, I. V. Sherstov, and V. A. Vasiliev, "High-sensitivity laser photoacoustic leak detector," Optical Engineering 46(6), 064302 (2007).

11. A. Miklós, P. Hess, and Z. Bozóki, "Application of acoustic resonators in photoacoustic trace gas analysis," Review of scientific instruments 72(4), 1937-1955 (2001).

12. V. Zéninari, R. Vallon, C. Risser, and B. Parvitte, "Photoacoustic detection of methane in large concentrations with a Helmholtz sensor: Simulation and experimentation", International Journal of Thermophysics 37(1), 7 (2016).

13. Y. V. Kistenev, A. I. Karapuzikov, N. Y. Kostyukova, M. K. Starikova, A. A. Boyko, E. B. Bukreeva, A. A. Bulanova, D. B. Kolker, D. A. Kuzmin, K. G. Zenov, and A. A. Karapuzikov, "Screening of patients with bronchopulmonary diseases using methods of infrared laser photoacoustic spectroscopy and principal component analysis," Journal of Biomedical Optics 20(6), 065001 (2015).

14. Yu V. Kistenev, A. V. Borisov, D. A. Kuzmin, O. V. Penkova, N. Y. Kostyukova, and A. A. Karapuzikov, "Exhaled air analysis using wideband wave number tuning range IR laser photoacoustic spectroscopy," Journal of Biomedical Optics 22(1), 017002 (2017).

15. J. Li , W. Chen, and B. Yu, "Recent Progress on Infrared Photoacoustic Spectroscopy Techniques," Applied Spectroscopy Reviews 46, 440-471 (2011).

16. D. D. Arslanov, M. P. P. Castro, N. A. Creemers, A. H. Neerincx, M. Spunei, J. Mandon, S. M. Cristescu, P. Merkus, and F. J. M. Harren, "Optical parametric oscillator-based photoacoustic detection of hydrogen cyanide for biomedical applications,” Journal of Biomedical Optics 18(10), 107002 (2013).

17. A. A. Karapuzikov, I. V. Sherstov, D. B. Kolker, A. I. Karapuzikov, Y. V. Kistenev, D. A. Kuzmin, M. Y. Shtyrov, N. Y. Dukhovnikova, K. G. Zenov, A. A. Boyko, M. K. Starikova, I. I. Tikhonyuk, I. B. Miroshnichenko, M. B. Miroshnichenko, Y. B. Myakishev, and V. N. Lokonov, "LaserBreeze gas analyzer for noninvasive diagnostics of air exhaled by patients," Physics of Wave Phenomena 22(3), 189-196 (2014).

18. L. Pomerantsev, O. Y. Rodionova, "Concept and role of extreme objects in PCA/SIMCA," Journal of Chemometrics 28(5), 429-438 (2014).

19. G. R. G. Lanckriet, N. Cristianini, P. Bartlett, L. El Ghaoui, and M. I. Jordan, "Learning the kernel matrix with semidefinite programming," Journal of Machine Learning Research 5, 27-72 (2004).

20. A. V. Borisov, Y. V. Kistenev, D. A. Kuzmin, V. V. Nikolaev, A. V. Shapovalov, and D. A. Vrazhnov, "Development of classification rules for a screening diagnostics of lung cancer patients based on the spectral analysis of metabolic profiles in the exhaled air," Proceedings of the Scientific-Practical Conference "Research and Development - 2016", 573-580 (2016). 